DESY 06-129

August, 2006

\title{
Measurement of azimuthal asymmetries in neutral current deep inelastic scattering at HERA
}

\author{
ZEUS Collaboration
}

\begin{abstract}
The distribution of the azimuthal angle of charged and neutral hadrons relative to the lepton plane has been studied for neutral current deep inelastic ep scattering using an integrated luminosity of $45 \mathrm{pb}^{-1}$ taken with the ZEUS detector. The kinematic range is $100<Q^{2}<8000 \mathrm{GeV}^{2}, 0.2<y<0.8$ and $0.01<x<0.1$ where $Q^{2}$ is the virtuality of the exchanged boson, $y$ is the inelasticity and $x$ is the Bjorken variable. The measurements were made in the hadronic centre-ofmass system. The analysis exploits the energy-flow method, which allows the measurement to be made over a larger range of pseudorapidity compared to previous results. The dependence of the moments of the azimuthal distributions on the pseudorapidity and minimum transverse energy of the final-state hadrons are presented. Although the predictions from next-to-leading-order QCD describe the data better than do the Monte Carlo models incorporating leading-logarithm parton showers, they still fail to describe the magnitude of the asymmetries. This suggests that higher-order calculations may be necessary to describe these data.
\end{abstract}




\section{The ZEUS Collaboration}

S. Chekanov, M. Derrick, S. Magill, S. Miglioranzi ${ }^{1}$, B. Musgrave, D. Nicholass ${ }^{1}$, J. Repond, R. Yoshida

Argonne National Laboratory, Argonne, Illinois 60439-4815, USA ${ }^{n}$

M.C.K. Mattingly

Andrews University, Berrien Springs, Michigan 49104-0380, USA

N. Pavel ${ }^{\dagger}$, A.G. Yagües Molina

Institut für Physik der Humboldt-Universität zu Berlin, Berlin, Germany

S. Antonelli, P. Antonioli, G. Bari, M. Basile, L. Bellagamba, M. Bindi, D. Boscherini, A. Bruni, G. Bruni, L. Cifarelli, F. Cindolo, A. Contin, M. Corradi², S. De Pasquale, G. Iacobucci, A. Margotti, R. Nania, A. Polini, L. Rinaldi, G. Sartorelli, A. Zichichi University and INFN Bologna, Bologna, Italy ${ }^{e}$

G. Aghuzumtsyan, D. Bartsch, I. Brock, S. Goers, H. Hartmann, E. Hilger, H.-P. Jakob, M. Jüngst, O.M. Kind, E. Paul ${ }^{3}$, J. Rautenberg ${ }^{4}$, R. Renner, U. Samson ${ }^{5}$, V. Schönberg, M. Wang, M. Wlasenko

Physikalisches Institut der Universität Bonn, Bonn, Germany ${ }^{b}$

N.H. Brook, G.P. Heath, J.D. Morris, T. Namsoo

H.H. Wills Physics Laboratory, University of Bristol, Bristol, United Kingdom ${ }^{m}$

M. Capua, S. Fazio, A. Mastroberardino, M. Schioppa, G. Susinno, E. Tassi

Calabria University, Physics Department and INFN, Cosenza, Italy ${ }^{e}$

J.Y. $\mathrm{Kim}^{6}$, K.J. $\mathrm{Ma}^{7}$

Chonnam National University, Kwangju, South Korea ${ }^{g}$

Z.A. Ibrahim, B. Kamaluddin, W.A.T. Wan Abdullah

Jabatan Fizik, Universiti Malaya, 50603 Kuala Lumpur, Malaysia ${ }^{r}$

Y. Ning, Z. Ren, F. Sciulli

Nevis Laboratories, Columbia University, Irvington on Hudson, New York 10027o

J. Chwastowski, A. Eskreys, J. Figiel, A. Galas, M. Gil, K. Olkiewicz, P. Stopa, L. Zawiejski

The Henryk Niewodniczanski Institute of Nuclear Physics, Polish Academy of Sciences, Cracow, Poland ${ }^{i}$

L. Adamczyk, T. Bołd, I. Grabowska-Bołd, D. Kisielewska, J. Łukasik, M. Przybycień,

L. Suszycki

Faculty of Physics and Applied Computer Science, AGH-University of Science and Technology, Cracow, Poland ${ }^{p}$ 
A. Kotański ${ }^{8}$, W. Słomiński

Department of Physics, Jagellonian University, Cracow, Poland

V. Adler, U. Behrens, I. Bloch, A. Bonato, K. Borras, N. Coppola, J. Fourletova, A. Geiser,

D. Gladkov, P. Göttlicher ${ }^{9}$, I. Gregor, O. Gutsche, T. Haas, W. Hain, C. Horn, B. Kahle,

U. Kötz, H. Kowalski, H. Lim¹0, E. Lobodzinska, B. Löhr, R. Mankel, I.-A. Melzer-

Pellmann, A. Montanari, C.N. Nguyen, D. Notz, A.E. Nuncio-Quiroz, R. Santamarta,

U. Schneekloth, A. Spiridonov ${ }^{11}$, H. Stadie, U. Stösslein, D. Szuba ${ }^{12}$, J. Szuba ${ }^{13}$, T. Theedt,

G. Watt, G. Wolf, K. Wrona, C. Youngman, W. Zeuner

Deutsches Elektronen-Synchrotron DESY, Hamburg, Germany

S. Schlenstedt

Deutsches Elektronen-Synchrotron DESY, Zeuthen, Germany

G. Barbagli, E. Gallo, P. G. Pelfer

University and INFN, Florence, Italy ${ }^{e}$

A. Bamberger, D. Dobur, F. Karstens, N.N. Vlasov ${ }^{14}$

Fakultät für Physik der Universität Freiburg i.Br., Freiburg i.Br., Germany ${ }^{b}$

P.J. Bussey, A.T. Doyle, W. Dunne, J. Ferrando, D.H. Saxon, I.O. Skillicorn

Department of Physics and Astronomy, University of Glasgow, Glasgow, United Kingdom $^{m}$

I. Gialas ${ }^{15}$

Department of Engineering in Management and Finance, Univ. of Aegean, Greece

T. Gosau, U. Holm, R. Klanner, E. Lohrmann, H. Salehi, P. Schleper, T. Schörner-Sadenius, J. Sztuk, K. Wichmann, K. Wick

Hamburg University, Institute of Exp. Physics, Hamburg, Germany ${ }^{b}$

C. Foudas, C. Fry, K.R. Long, A.D. Tapper

Imperial College London, High Energy Nuclear Physics Group, London, United Kingdom ${ }^{m}$

M. Kataoka ${ }^{16}$, T. Matsumoto, K. Nagano, K. Tokushuku ${ }^{17}$, S. Yamada, Y. Yamazaki Institute of Particle and Nuclear Studies, KEK, Tsukuba, Japan ${ }^{f}$

A.N. Barakbaev, E.G. Boos, A. Dossanov, N.S. Pokrovskiy, B.O. Zhautykov

Institute of Physics and Technology of Ministry of Education and Science of Kazakhstan, Almaty, Kazakhstan

D. Son

Kyungpook National University, Center for High Energy Physics, Daegu, South Korea ${ }^{g}$ 
J. de Favereau, K. Piotrzkowski

Institut de Physique Nucléaire, Université Catholique de Louvain, Louvain-la-Neuve, Belgium $^{q}$

F. Barreiro, C. Glasman ${ }^{18}$, M. Jimenez, L. Labarga, J. del Peso, E. Ron, J. Terrón, M. Zambrana

Departamento de Física Teórica, Universidad Autónoma de Madrid, Madrid, Spain ${ }^{l}$

F. Corriveau, C. Liu, R. Walsh, C. Zhou

Department of Physics, McGill University, Montréal, Québec, Canada H3A 2T8 ${ }^{a}$

T. Tsurugai

Meiji Gakuin University, Faculty of General Education, Yokohama, Japan ${ }^{f}$

A. Antonov, B.A. Dolgoshein, I. Rubinsky, V. Sosnovtsev, A. Stifutkin, S. Suchkov Moscow Engineering Physics Institute, Moscow, Russia ${ }^{j}$

R.K. Dementiev, P.F. Ermolov, L.K. Gladilin, I.I. Katkov, L.A. Khein, I.A. Korzhavina, V.A. Kuzmin, B.B. Levchenko ${ }^{19}$, O.Yu. Lukina, A.S. Proskuryakov, L.M. Shcheglova, D.S. Zotkin, S.A. Zotkin

Moscow State University, Institute of Nuclear Physics, Moscow, Russia ${ }^{k}$

I. Abt, C. Büttner, A. Caldwell, D. Kollar, W.B. Schmidke, J. Sutiak

Max-Planck-Institut für Physik, München, Germany

G. Grigorescu, A. Keramidas, E. Koffeman, P. Kooijman, A. Pellegrino, H. Tiecke, M. Vázquez ${ }^{20}$, L. Wiggers

NIKHEF and University of Amsterdam, Amsterdam, Netherlands ${ }^{h}$

N. Brümmer, B. Bylsma, L.S. Durkin, A. Lee, T.Y. Ling

Physics Department, Ohio State University, Columbus, Ohio $43210^{n}$

P.D. Allfrey, M.A. Bell, A.M. Cooper-Sarkar, A. Cottrell, R.C.E. Devenish, B. Foster, C. Gwenlan ${ }^{21}$, K. Korcsak-Gorzo, S. Patel, V. Roberfroid ${ }^{22}$, A. Robertson, P.B. Straub, C. Uribe-Estrada, R. Walczak

Department of Physics, University of Oxford, Oxford United Kingdom ${ }^{m}$

P. Bellan, A. Bertolin, R. Brugnera, R. Carlin, R. Ciesielski, F. Dal Corso, S. Dusini,

A. Garfagnini, S. Limentani, A. Longhin, L. Stanco, M. Turcato

Dipartimento di Fisica dell' Università and INFN, Padova, Italy ${ }^{e}$

B.Y. Oh, A. Raval, J. Ukleja ${ }^{23}$, J.J. Whitmore

Department of Physics, Pennsylvania State University, University Park, Pennsylvania $16800^{\circ}$

Y. Iga

Polytechnic University, Sagamihara, Japan ${ }^{f}$ 
G. D’Agostini, G. Marini, A. Nigro

Dipartimento di Fisica, Università 'La Sapienza' and INFN, Rome, Italy ${ }^{e}$

J.E. Cole, J.C. Hart

Rutherford Appleton Laboratory, Chilton, Didcot, Oxon, United Kingdom ${ }^{m}$

H. Abramowicz ${ }^{24}$, A. Gabareen, R. Ingbir, S. Kananov, A. Levy

Raymond and Beverly Sackler Faculty of Exact Sciences, School of Physics, Tel-Aviv

University, Tel-Aviv, Israel ${ }^{d}$

M. Kuze

Department of Physics, Tokyo Institute of Technology, Tokyo, Japan ${ }^{f}$

R. Hori, S. Kagawa ${ }^{25}$, S. Shimizu, T. Tawara

Department of Physics, University of Tokyo, Tokyo, Japan ${ }^{f}$

R. Hamatsu, H. Kaji, S. Kitamura ${ }^{26}$, O. Ota, Y.D. Ri

Tokyo Metropolitan University, Department of Physics, Tokyo, Japan ${ }^{f}$

M.I. Ferrero, V. Monaco, R. Sacchi, A. Solano

Università di Torino and INFN, Torino, Italy ${ }^{e}$

M. Arneodo, M. Ruspa

Università del Piemonte Orientale, Novara, and INFN, Torino, Italy ${ }^{e}$

S. Fourletov, J.F. Martin

Department of Physics, University of Toronto, Toronto, Ontario, Canada M5S 1A7 a

S.K. Boutle ${ }^{15}$, J.M. Butterworth, R. Hall-Wilton ${ }^{20}$, T.W. Jones, J.H. Loizides, M.R. Sutton ${ }^{27}$,

C. Targett-Adams, M. Wing

Physics and Astronomy Department, University College London, London, United Kingdom ${ }^{m}$

B. Brzozowska, J. Ciborowski ${ }^{28}$, G. Grzelak, P. Kulinski, P. Łużniak ${ }^{29}$, J. Malka ${ }^{29}$, R.J. Nowak, J.M. Pawlak, T. Tymieniecka, A. Ukleja ${ }^{30}$, A.F. Żarnecki

Warsaw University, Institute of Experimental Physics, Warsaw, Poland

M. Adamus, P. Plucinski ${ }^{31}$

Institute for Nuclear Studies, Warsaw, Poland

Y. Eisenberg, I. Giller, D. Hochman, U. Karshon, M. Rosin

Department of Particle Physics, Weizmann Institute, Rehovot, Israel ${ }^{c}$

E. Brownson, T. Danielson, A. Everett, D. Kçira, D.D. Reeder, P. Ryan, A.A. Savin,

W.H. Smith, H. Wolfe

Department of Physics, University of Wisconsin, Madison, Wisconsin 53706, USA ${ }^{n}$ 
S. Bhadra, C.D. Catterall, Y. Cui, G. Hartner, S. Menary, U. Noor, M. Soares, J. Standage, J. Whyte

Department of Physics, York University, Ontario, Canada M3J 1P3 ${ }^{a}$ 
1 also affiliated with University College London, UK

2 also at University of Hamburg, Germany, Alexander von Humboldt Fellow

${ }^{3}$ retired

${ }^{4}$ now at Univ. of Wuppertal, Germany

${ }^{5}$ formerly U. Meyer

${ }^{6}$ supported by Chonnam National University in 2005

${ }^{7}$ supported by a scholarship of the World Laboratory Björn Wiik Research Project

${ }^{8}$ supported by the research grant no. 1 P03B 04529 (2005-2008)

${ }^{9}$ now at DESY group FEB, Hamburg, Germany

${ }^{10}$ now at Argonne National Laboratory, Argonne, IL, USA

11 also at Institut of Theoretical and Experimental Physics, Moscow, Russia

12 also at INP, Cracow, Poland

13 on leave of absence from FPACS, AGH-UST, Cracow, Poland

14 partly supported by Moscow State University, Russia

15 also affiliated with DESY

${ }^{16}$ now at ICEPP, University of Tokyo, Japan

17 also at University of Tokyo, Japan

18 Ramón y Cajal Fellow

19 partly supported by Russian Foundation for Basic Research grant no. 05-02-39028NSFC-a

20 now at CERN, Geneva, Switzerland

${ }^{21}$ PPARC Postdoctoral Research Fellow

22 EU Marie Curie Fellow

23 partially supported by Warsaw University, Poland

24 also at Max Planck Institute, Munich, Germany, Alexander von Humboldt Research Award

${ }^{25}$ now at KEK, Tsukuba, Japan

${ }^{26}$ Department of Radiological Science

27 PPARC Advanced fellow

28 also at Eódź University, Poland

${ }^{29}$ Łódź University, Poland

30 supported by the Polish Ministry for Education and Science grant no. 1 P03B 12629

31 supported by the Polish Ministry for Education and Science grant no. 1 P03B 14129

$\dagger$ deceased 
a supported by the Natural Sciences and Engineering Research Council of Canada (NSERC)

$b$ supported by the German Federal Ministry for Education and Research (BMBF), under contract numbers HZ1GUA 2, HZ1GUB 0, HZ1PDA 5, HZ1VFA 5

$c$ supported in part by the MINERVA Gesellschaft für Forschung GmbH, the Israel Science Foundation (grant no. 293/02-11.2) and the U.S.-Israel Binational Science Foundation

$d$ supported by the German-Israeli Foundation and the Israel Science Foundation

$e$ supported by the Italian National Institute for Nuclear Physics (INFN)

${ }^{f} \quad$ supported by the Japanese Ministry of Education, Culture, Sports, Science and Technology (MEXT) and its grants for Scientific Research

$g$ supported by the Korean Ministry of Education and Korea Science and Engineering Foundation

$h \quad$ supported by the Netherlands Foundation for Research on Matter (FOM)

$i$ supported by the Polish State Committee for Scientific Research, grant no. 620/E-77/SPB/DESY/P-03/DZ 117/2003-2005 and grant no. 1P03B07427/2004-2006

$j$ partially supported by the German Federal Ministry for Education and Research (BMBF)

$k$ supported by RF Presidential grant N 1685.2003.2 for the leading scientific schools and by the Russian Ministry of Education and Science through its grant for Scientific Research on High Energy Physics

$l$ supported by the Spanish Ministry of Education and Science through funds provided by CICYT

$m$ supported by the Particle Physics and Astronomy Research Council, UK

$n$ supported by the US Department of Energy

$o$ supported by the US National Science Foundation

$p$ supported by the Polish Ministry of Scientific Research and Information Technology, grant no. 112/E-356/SPUB/DESY/P-03/DZ 116/2003-2005 and 1 P03B 06527

$q$ supported by FNRS and its associated funds (IISN and FRIA) and by an Inter-University Attraction Poles Programme subsidised by the Belgian Federal Science Policy Office

$r$ supported by the Malaysian Ministry of Science, Technology and Innovation/Akademi Sains Malaysia grant SAGA 66-02-03-0048 


\section{Introduction}

The description of the hadronic final state in deep inelastic scattering (DIS) is influenced by perturbative Quantum Chromodynamics (pQCD) in several ways that can be calculated through exact matrix elements or leading-logarithm parton showers. Measurements of the azimuthal distribution of hadrons in the semi-inclusive process $e+p \rightarrow e+h+X$ in DIS are sensitive to predictions of $\mathrm{pQCD}$. The azimuthal angle, $\phi$, is defined in the hadronic centre-of-mass (HCM) frame as the angle between the hadron-production plane and the lepton-scattering plane as illustrated in Fig. 1.

Asymmetries in $\phi$ result from the final-state hadrons having transverse momentum with respect to the colliding virtual photon and the incoming proton. In $\mathrm{pQCD}, \alpha_{s}$-order QCD processes such as QCD Compton (QCDC) $\left(\gamma^{*} q \rightarrow q g\right)$ and boson-gluon fusion (BGF) $\left(\gamma^{*} g \rightarrow q \bar{q}\right)$ are the main sources of these hadrons. These two processes have different $\phi$ behaviours [1] as well as a different pseudorapidity, $\eta$, dependence, defined here with respect to the incoming proton direction in the HCM frame. Figure 2(a) shows that hadrons from BGF and QCDC dominate over quark-parton-model (QPM) $\left(\gamma^{*} q \rightarrow q\right)$ events in the region $-4<\eta^{\mathrm{HCM}}<0$. In addition, gluons and quarks from the QCDC process have different pseudorapidity dependencies, as illustrated in Fig. 2(b).

The azimuthal dependence for semi-inclusive neutral current (NC) DIS can be written [25] as:

$$
\frac{d \sigma^{e p \rightarrow e h X}}{d \phi}=\mathcal{A}+\mathcal{B} \cos \phi+\mathcal{C} \cos 2 \phi+\mathcal{D} \sin \phi+\mathcal{E} \sin 2 \phi
$$

The azimuthal asymmetries, specified by the parameters $\mathcal{B}, \mathcal{C}, \mathcal{D}$ and $\mathcal{E}$, are extracted from the data by calculating the statistical moments of the experimental distributions:

$$
\begin{aligned}
\langle\cos \phi\rangle & =\frac{\mathcal{B}}{2 \mathcal{A}} ; & \langle\sin \phi\rangle & =\frac{\mathcal{D}}{2 \mathcal{A}} \\
\langle\cos 2 \phi\rangle & =\frac{\mathcal{C}}{2 \mathcal{A}} ; & \langle\sin 2 \phi\rangle & =\frac{\mathcal{E}}{2 \mathcal{A}}
\end{aligned}
$$

Equation (1) results from the polarisation of the exchanged virtual photon. The coefficient $\mathcal{B}$ originates from the interference between the transversely and longitudinally polarised components; the coefficient $\mathcal{C}$ is due to the interference of amplitudes corresponding to the +1 and -1 helicity parts of the transversely polarised exchanged boson. The coefficients $\mathcal{D}$ and $\mathcal{E}$ arise from parity-violating weak interactions or longitudinal polarisation of the initial lepton beam [3]. They vanish for purely electromagnetic interactions with unpolarised beams. 
It has been proposed [4] to analyse the asymmetry as a function of the transverse momentum cutoff, $p_{T}^{\text {cut }}$, of a detected hadron. Such a cut is efficient in removing QPM events. Consequently, at higher $p_{T}^{\text {cut }}$ values a better agreement should be obtained with the perturbative QCD predictions. A model using a resummation formalism [6] to predict azimuthal asymmetries has also been proposed. This model predicts that logarithmic corrections due to soft parton emission could be large. A recent paper [7] showed that a part of the asymmetries previously measured by the ZEUS collaboration $[8,9]$ may come from terms that are not included in the perturbative gluon radiation but are related to the intrinsic transverse motion of quarks.

For NC DIS with an unpolarised lepton beam, the $\langle\cos \phi\rangle$ and $\langle\cos 2 \phi\rangle$ values have been measured by the ZEUS collaboration $[8,9]$ to be at the few percent level. The first publication [8] measured the azimuthal distribution for charged hadrons, whereas the second [9] was performed for jets of high transverse energy. The present analysis used the energy-flow method, which permits both neutral and charged hadrons to be included in the measurements. This analysis was performed using a similar data sample but in an extended kinematic range compared to previous publications. In particular, the polarangle range of the measurements was increased with respect to the previous studies $[8,9]$. The energy-flow method enhances the contribution of leading hadrons since the direction of each particle in the final state is weighted with its transverse energy $[10,11]$. This method is discusssed in detail elsewhere [12]. Additionally, the values $\langle\sin \phi\rangle$ and $\langle\sin 2 \phi\rangle$ were determined, although they are expected $[3,5]$ to be much smaller than $\langle\cos \phi\rangle$ and $\langle\cos 2 \phi\rangle$.

This paper presents measurements of $\langle\cos \phi\rangle,\langle\cos 2 \phi\rangle,\langle\sin \phi\rangle$ and $\langle\sin 2 \phi\rangle$ as a function of the pseudorapidity, $\eta^{\mathrm{HCM}}$, and minimum transverse energy, $E_{T, \text { min }}^{\mathrm{HCM}}\left(\right.$ instead of $p_{T}^{\text {cut }}[4]$ ), of the final-state hadrons. The results are compared to theoretical expectations.

\section{Data sample}

The experimental results are based on the data collected in 1995-97 with the ZEUS detector at HERA. Protons of $820 \mathrm{GeV}$ collided with $27.5 \mathrm{GeV}$ unpolarised ${ }^{1}$ positrons. Neutral current DIS events were selected from data corresponding to an integrated luminosity of $45 \mathrm{pb}^{-1}$. A detailed description of the ZEUS detector can be found elsewhere [14]. A brief outline of the components that are most relevant for this analysis is given below.

The high-resolution uranium-scintillator calorimeter (CAL) [15] consists of three parts: the forward (FCAL), the barrel (BCAL) and the rear (RCAL) calorimeters. Each part

\footnotetext{
${ }^{1}$ The positrons were transversely polarised due to the Sokolov-Ternov [13] effect, but had no longitudinal polarisation.
} 
is subdivided transversely into towers and longitudinally into one electromagnetic section (EMC) and either one (in RCAL) or two (in BCAL and FCAL) hadronic sections (HAC). The smallest subdivision of the calorimeter is called a cell. The CAL energy resolutions, as measured under test-beam conditions, are $\sigma(E) / E=0.18 / \sqrt{E}$ for electrons and $\sigma(E) / E=0.35 / \sqrt{E}$ for hadrons, with $E$ in $\mathrm{GeV}$.

Charged particles are tracked in the central tracking detector (CTD) [16], which operates in a magnetic field of $1.43 \mathrm{~T}$ provided by a thin superconducting coil. The CTD consists of 72 cylindrical drift chamber layers, organised in 9 superlayers covering the polar-angle ${ }^{2}$ region $15^{\circ}<\theta<164^{\circ}$. The transverse-momentum resolution for full-length tracks is $\sigma\left(p_{T}\right) / p_{T}=0.0058 p_{T} \oplus 0.0065 \oplus 0.0014 / p_{T}$, with $p_{T}$ in $\mathrm{GeV}$.

The selection criteria were based on an earlier ZEUS investigation [8]. The main requirements on the event were:

- an identified scattered positron with energy $E_{e}^{\prime}>10 \mathrm{GeV}$. Energy deposits in the CAL, consistent with being a photon, within an $\eta-\phi$ cone of radius 1 around the direction of the candidate positron, were removed from the calculation of the positron energy;

- $100<Q^{2}<8000 \mathrm{GeV}^{2}, 0.2<y<0.8$ and $0.01<x<0.1$. The quantities $x, y$ and $Q^{2}$ are respectively $x$-Bjorken, the inelasticity, and the negative square of the exchanged boson virtuality. The double angle method was used to reconstruct these variables and so determine the direction of the exchanged boson [17]. The mean values of $x, y$ and $Q^{2}$ were respectively $0.0222,0.351$ and $700 \mathrm{GeV}^{2}$. The final sample consisted of 16472 events.

Charged and neutral final-state particles were reconstructed using a combination of track and calorimeter information that optimises the resolution of the reconstructed kinematic variables [18]. The selected tracks and calorimeter clusters are referred to as energy flow objects (EFOs). The EFOs were required to satisfy the following:

- transverse momentum in the laboratory frame $p_{T}>0.15 \mathrm{GeV}$;

- polar angle $\theta>8^{\circ}$.

These cuts ensured the analysis was performed in a region of high acceptance and high detector efficiency. An average of about 18 EFOs per event were reconstructed, yielding a total of 293000 objects, each of which provided a value of the azimuthal angle, $\phi$, used for further analysis.

\footnotetext{
${ }^{2}$ The ZEUS coordinate system is a right-handed Cartesian system, with the $Z$ axis pointing in the proton beam direction, referred to as the "forward direction", and the $X$ axis pointing left towards the centre of HERA. The coordinate origin is at the nominal interaction point.
} 


\section{Method}

The energy-flow method was proposed $[19,20]$ to ensure that the inclusive variables such as those measured here are infra-red and collinear safe due to the direction of each particle being weighted by its transverse energy. In addition, the energy flow method has the experimental advantage of higher statistics and probing a larger region of phase space compared to using charged particles only [8] or reconstructing jets [9].

In this analysis, the moments of trigonometric functions of the azimuthal angle are calculated as follows. For a function, $F\left(n \phi^{\mathrm{HCM}}\right)$, where $F\left(n \phi^{\mathrm{HCM}}\right)$ can be $\sin \left(n \phi^{\mathrm{HCM}}\right)$ or $\cos \left(n \phi^{\mathrm{HCM}}\right)$ and $n=1$ or 2 , the mean value was determined using the formula:

$$
\left\langle F\left(n \phi^{\mathrm{HCM}}\right)\right\rangle=\frac{\sum_{i} E_{T, i}^{\mathrm{HCM}} F\left(n \phi_{i}^{\mathrm{HCM}}\right)}{\sum_{i} E_{T, i}^{\mathrm{HCM}}},
$$

where $E_{T}^{\mathrm{HCM}}$ is the transverse energy and $\phi$ the azimuthal angle for each EFO, $i$. The value is calculated in bins of $\eta^{\mathrm{HCM}}$, semi-inclusively, and for different minimum cuts on the transverse energy of the EFO, $E_{T, \text { min }}^{\mathrm{HCM}}$. Equation (2) is also used for the determination of the moments in theoretical calculations, in which the sum is either over final-state hadrons or partons. The mean values are not expected to be sensitive to uncertainties in fragmentation functions and calorimeter energy scale, since such effects contribute to both the numerator and the denominator.

\section{Correction procedure}

Monte Carlo (MC) events were used to correct the data for detector acceptances. For all generated events, the ZEUS detector response was simulated in detail using a program based on GEANT 3.13 [21].

Neutral current events with electroweak radiative corrections were simulated with the LePto 6.5.1 [22] program interfaced to Heracles 4.6.1 [23, 24] via the DJANGoH 1.1 program $[25,26]$. High-order QCD processes were simulated using the MEPS option of LePto. A second sample of MC events was generated with ArIAdne 4.12 [27], where the QCD cascade is simulated with the colour-dipole model. In all cases, the events were generated using the CTEQ4D parton density parametrisation [28] of the proton. The final-state partonic system was hadronised using the Lund string model as implemented in JETSET 7.4 [29].

For a given bin, $j$, in $\eta^{\mathrm{HCM}}$ and $F\left(n \phi^{\mathrm{HCM}}\right)$, two correction factors for $F\left(n \phi^{\mathrm{HCM}}\right)$ were derived from $\mathrm{MC}$ as the ratio of values determined using hadrons and EFOs: 


$$
C_{j}=\frac{\left[\sum_{i} E_{T, i}^{\mathrm{HCM}} F\left(n \phi_{i}^{\mathrm{HCM}}\right)\right]_{\mathrm{had}, j}^{\mathrm{MC}}}{\left[\sum_{i} E_{T, i}^{\mathrm{HCM}} F\left(n \phi_{i}^{\mathrm{HCM}}\right)\right]_{\mathrm{EFO}, j}^{\mathrm{MC}}}, \quad D_{j}=\frac{\left[\sum_{i} E_{T, i}^{\mathrm{HCM}}\right]_{\mathrm{had}, j}^{\mathrm{MC}}}{\left[\sum_{i} E_{T, i}^{\mathrm{HCM}}\right]_{\mathrm{EFO}, j}^{\mathrm{MC}}},
$$

where $C_{j}$ corrects the trigonometric function weighted with $E_{T}$ and $D_{j}$ corrects the sum of $E_{T}$ in the bin, $j$. The corrected data for a bin in $\eta^{\mathrm{HCM}}$ is then given by:

$$
\left\langle F\left(n \phi^{\mathrm{HCM}}\right)\right\rangle_{\mathrm{corr}}=\frac{\sum_{j}\left(\sum_{i} E_{T, i}^{\mathrm{HCM}} F\left(n \phi_{i}^{\mathrm{HCM}}\right)\right)_{\mathrm{EFO}, j} \cdot C_{j}}{\sum_{j}\left(\sum_{i} E_{T, i}^{\mathrm{HCM}}\right)_{\mathrm{EFO}, j} \cdot D_{j}} .
$$

The overall correction factors are about $10 \%$ and arise mainly from undetected hadrons. For this approach to be valid, the uncorrected energy flow in the data must be well described by the MC simulations at the detector level. This condition was satisfied [30] by both the LEPTO and ARIADNE simulations in the $\eta^{\mathrm{HCM}}$ and $E_{T}^{\mathrm{HCM}}$ regions under investigation. The samples of LEPTO events were used for the final corrections.

Systematic uncertainties of the azimuthal asymmetry were determined by varying the event selection cuts within their reconstruction resolution and the total systematic uncertainty was taken as the sum in quadrature of the individual systematics. The dominant contributions originated from the following sources (maximal deviations for $\langle\cos \phi\rangle$ are shown in parentheses):

- the use of the ARIADNE MC model to correct the data (0.017);

- varying the cut on the $p_{T}$ of the final-state objects from $0.15 \mathrm{GeV}$ to $0.2 \mathrm{GeV}$ to estimate the effect due to low- $p_{T}$ tracks $(0.013)$;

- the inclusion of energy deposits consistent with being a photon for the calculation of the hadronic angle, used in the double angle method [17], and therefore the transformation to the HCM frame (0.009).

The effect of the variation of other selection cuts was negligible.

\section{QCD calculations}

The data were compared to the MC programs LePto and ARIADnE, described in the previous section, and to a next-to-leading-order (NLO) prediction. In these two MC programs, the azimuthal-angular distribution is implemented according to the first-order QCD matrix elements for QCDC and BGF. The parton-shower and soft-matrix-element events do not introduce azimuthal asymmetries. The NLO predictions were calculated using the dipole factorisation formulae [31] implemented in the DisENT program [31, 
32]. The calculations used a generalised version of the subtraction method [33] and were performed in the massless $\overline{\mathrm{MS}}$ renormalisation and factorisation schemes. The azimuthalangle distribution is calculated to NLO by the DisENT program through the use of the photon leptonic current in the amplitudes for the QCDC, BGF and $\mathcal{O}\left(\alpha_{s}^{2}\right)$ processes. This program contains neither $Z^{0}$ exchange nor hadronisation effects. The following settings were used as defaults for DISENT: the number of flavours was five, the factorisation and renormalisation scales were $\mu_{F, R}=Q$, and the parton distribution function was CTEQ3M [34]. Samples of events from LEPTO 6.5.1 were used to correct the NLO QCD calculations for $Z^{0}$-exchange effects, hadronisation and undetected particles due to the requirements on $p_{T}$ and $\theta$ of the produced hadrons. The corrections were performed using the formalism in Section 4 by replacing EFOs by partons with the $C_{j}$ and $D_{j}$ factor estimated from LEPTO 6.5.1.

The uncertainty in the DISENT predictions was estimated by changing the following:

- the renormalisation and factorisation scales were individually changed to $\mu_{F, R}=Q / 2$ and $2 Q$;

- the parton distribution functions CTEQ4M [28], CTEQ5M [35], were used;

- the correction for $Z^{0}$-exchange effects, hadronisation and undetected particles was repeated with the ARIADNE 4.12 MC.

The above uncertainties were at most 0.004 in both $\langle\cos \phi\rangle$ and $\langle\cos 2 \phi\rangle$. They were added in quadrature and are displayed in the figures as shaded bands around the central prediction. The sensitivity to different gluon distributions was checked by using the MRST99 [36] parton distributions functions with an increased or decreased gluon density; the differences were negligible.

\section{$6 \quad$ Results}

Azimuthal asymmetries have been measured in NC DIS events with the requirements: $100<Q^{2}<8000 \mathrm{GeV}^{2}, 0.2<y<0.8$ and $0.01<x<0.1$ for hadrons with $p_{T}>0.15 \mathrm{GeV}$ and $\theta>8^{\circ}$. The mean values of $\cos \phi^{\mathrm{HCM}}, \cos 2 \phi^{\mathrm{HCM}}, \sin \phi^{\mathrm{HCM}}$ and $\sin 2 \phi^{\mathrm{HCM}}$ are shown in Fig. 3 and given in Tables 1 and 2 as a function of $\eta^{\mathrm{HCM}}$. The data are compared with predictions from MC models and from NLO QCD as described in the previous section.

Figure 3 shows that the value of $\left\langle\cos \phi^{\mathrm{HCM}}\right\rangle$ is negative for $\eta^{\mathrm{HCM}}<-2$ but becomes positive for larger $\eta^{\mathrm{HCM}}$. This is in disagreement with both MC predictions, which are less negative for $\eta^{\mathrm{HCM}}<-2$ and remain negative for larger $\eta^{\mathrm{HCM}}$. The measured $\left\langle\cos 2 \phi^{\mathrm{HCM}}\right\rangle$ values are consistent with zero for $\eta^{\mathrm{HCM}}<-2$ but are positive for higher values of $\eta^{\mathrm{HCM}}$. This is consistent with the expectations from both LEPTO and ARIADNE. 
The NLO QCD predictions, corrected for hadronisation, agree better with the experimental values for $\left\langle\cos \phi^{\mathrm{HCM}}\right\rangle$ than do the MC predictions. The predictions from NLO QCD are more negative for $\eta^{\mathrm{HCM}}<-2$ than those from the MC generators and also have positive values for larger $\eta^{\mathrm{HCM}}$. However, the $\mathrm{NLO}$ calculation still fails to describe the magnitude of the asymmetry in the data. The comparison with NLO QCD was also made at higher $p_{T}$, greater than $1 \mathrm{GeV}$ (not shown). The Monte Carlo was used to correct the NLO for this cut; although the final correction for $\left\langle\cos \phi^{\mathrm{HCM}}\right\rangle$ was small, the correction for hadron removal was large. However, the comparison with the data was qualitatively the same as when the more inclusive cut was used. The disagreement between data and NLO suggests that higher-order calculations may be necessary to describe this distribution fully. Inclusion of higher orders through a resummation of large logarithmic terms is expected [6] to give an improved description compared to that of LO for $-5<\eta<-3$. However, the description is not significantly better than for the other predictions. For $\left\langle\cos 2 \phi^{\mathrm{HCM}}\right\rangle$, the NLO and MC predictions are similar and describe the data reasonably well.

Figure 3 shows that the values of $\left\langle\sin \phi^{\mathrm{HCM}}\right\rangle$ and $\left\langle\sin 2 \phi^{\mathrm{HCM}}\right\rangle$ are small. A deviation of $\left\langle\sin \phi^{\mathrm{HCM}}\right\rangle$ from zero at the level of three standard deviations is observed. The mean values are expected to be at least an order of magnitude smaller than the $\left\langle\cos \phi^{\mathrm{HCM}}\right\rangle$ term [5]. The values of $\left\langle\sin 2 \phi^{\mathrm{HCM}}\right\rangle$ are consistent with zero. None of the theoretical models include predictions for $\left\langle\sin \phi^{\mathrm{HCM}}\right\rangle$ or $\left\langle\sin 2 \phi^{\mathrm{HCM}}\right\rangle$.

To investigate the effect of the minimum transverse energy cut, $E_{T, \min }^{\mathrm{HCM}}$, on the asymmetries, the event sample was subdivided into three regions of $\eta^{\mathrm{HCM}}:-5<\eta^{\mathrm{HCM}}<-2.5$, $-2.5<\eta^{\mathrm{HCM}}<-1$ and $-1<\eta^{\mathrm{HCM}}<0$. For $E_{T, \text { min }}^{\mathrm{HCM}}=1 \mathrm{GeV}$, the acceptance is approximately $100 \%$. Below this value, some hadrons are removed by the $p_{T}>0.15 \mathrm{GeV}$ requirement mainly in the region $-2.5<\eta^{\mathrm{HCM}}<-1$. The data are shown in Fig. 4 , and given in Tables 3 and 4, compared to the predictions from LEPTO and ARIADNE MCs. As stated previously, NLO QCD predictions for higher $E_{T, \text { min }}^{\mathrm{HCM}}$ have large corrections for hadron removal.

The first region $-5<\eta^{\mathrm{HCM}}<-2.5$ is part of the current region in DIS defined in the Breit frame as $\eta^{\text {Breit }} \approx \eta^{\mathrm{HCM}}+2<0$; in this region the main contribution to the azimuthal asymmetry comes from QCDC and arises from hadrons from quark fragmentation (Fig. 2). This region was investigated in the first ZEUS analysis of azimuthal asymmetries [8] using charged hadrons. The data from the current analysis, shown in Fig. 4, confirm, with higher experimental precision and to higher $E_{T, \min }^{\mathrm{HCM}}$, that the value of $\left\langle\cos \phi^{\mathrm{HCM}}\right\rangle$ is more negative than expected from MC predictions. The $\left\langle\cos 2 \phi^{\mathrm{HCM}}\right\rangle$ values are small and consistent with zero and also in agreement with both LEPTO and ARIADnE.

The region $-2.5<\eta^{\mathrm{HCM}}<-1$ is still dominated by QCDC events but the contribution from BGF events increases. The ZEUS analysis of azimuthal asymmetries measured 
using jets [9] was based on hadrons from this region of phase space. In that analysis, a large positive value of $\left\langle\cos 2 \phi^{\mathrm{HCM}}\right\rangle$ was measured, whereas the $\left\langle\cos \phi^{\mathrm{HCM}}\right\rangle$ value was consistent with zero. The $\left\langle\cos 2 \phi^{\mathrm{HCM}}\right\rangle$ values agreed with the NLO QCD prediction and were inconsistent with the LO prediction. The results presented here in Fig. 4 confirm, with higher experimental precision, a small value of $\left\langle\cos \phi^{\mathrm{HCM}}\right\rangle$ and positive values for $\left\langle\cos 2 \phi^{\mathrm{HCM}}\right\rangle$ for all $E_{T, \mathrm{Hin}}^{\mathrm{HCM}}$. The MC predictions of LEPTO and ARIADNE are in good agreement with the data.

The third region, $-1<\eta^{\mathrm{HCM}}<0$, is populated roughly equally by hadrons from QCDC and from BGF processes. This measurement extends the kinematic region over those presented previously. The results are presented in Fig. 4. The $\left\langle\cos \phi^{\mathrm{HCM}}\right\rangle$ values are positive, contrary to MC predictions, whereas the $\left\langle\cos 2 \phi^{\mathrm{HCM}}\right\rangle$ values are positive and in agreement with $\mathrm{MC}$ predictions. These trends persist up to the highest $E_{T, \text { min }}^{\mathrm{HCM}}$ values measured.

\section{Summary and conclusions}

The azimuthal asymmetries in deep inelastic scattering have been measured in the hadronic centre-of-mass frame at HERA for a selected sample of neutral current events with $100<Q^{2}<8000 \mathrm{GeV}^{2}, 0.2<y<0.8$ and $0.01<x<0.1$. An energy-flow analysis method was used, which permitted the use of both neutral and charged hadrons and extends the phase space over previous measurements.

Azimuthal asymmetries have been investigated as a function of hadron pseudorapidity, $\eta^{\mathrm{HCM}}$. The value of $\left\langle\cos \phi^{\mathrm{HCM}}\right\rangle$ is negative for $\eta^{\mathrm{HCM}}<-2$ but becomes positive for larger $\eta^{\mathrm{HCM}}$. The distribution is not well described by the MC predictions of ARIADNE and LePTo. Although the predictions from NLO QCD describe the data better than do the MCs, they still fail to describe the magnitude of the asymmetries. This suggests that higher-order calculations may be necessary to describe these data. However, the predicted values of $\left\langle\cos 2 \phi^{\mathrm{HCM}}\right\rangle$ in both MC models and in NLO QCD agree with the data. A deviation of $\left\langle\sin \phi^{\mathrm{HCM}}\right\rangle$ from zero at the level of three standard deviations is observed. The values of $\left\langle\sin 2 \phi^{\mathrm{HCM}}\right\rangle$ are consistent with zero.

\section{Acknowledgements}

We thank the DESY Directorate for their strong support and encouragement. The effort of the HERA machine group is gratefully acknowledged. We thank the DESY computing and network services for their support as well as the engineers and technicians for their 
work in constructing and maintaining the ZEUS detector. Special thanks go to Thomas Gehrmann, Gunnar Ingelman, Krzysztof Kurek, Piet Mulders and Pavel Nadolsky for valuable discussions. 


\section{References}

[1] V. Hedberg et al., Proc. Workshop on Physics at HERA, W. Buchmüller and G. Ingelman (eds.), Vol. 1, p. 331. Hamburg, Germany, DESY (1992).

[2] A. Méndez, Nucl. Phys. B 145, 199 (1978).

[3] K. Hagiwara, K.-I. Hikasa and N. Kai, Phys. Rev. D 27, 84 (1983).

[4] J. Chay, S.D. Ellis and W.J. Stirling, Phys. Lett. B 269, 175 (1991).

[5] M. Ahmed and T. Gehrmann, Phys. Lett. B 465, 297 (1999).

[6] P.M. Nadolsky, D.R. Strump and C.-P. Yuan, Phys. Lett. B 515, 175 (2001).

[7] V. Barone, Z. Lu and B.-Q. Ma, Phys. Lett. B 632, 277 (2006).

[8] ZEUS Collab., J. Breitweg et al., Phys. Lett. B 481, 199 (2000).

[9] ZEUS Collab., S. Chekanov et al., Phys. Lett. B 551, 226 (2003).

[10] ZEUS Collab., M. Derrick et al., Z. Phys. C 59, 231 (1993);

ZEUS Collab., M. Derrick et al., Phys. Lett. B 338, 483 (1994);

ZEUS Collab., S. Chekanov et al., Phys. Rev. D 65, 052001 (2002).

[11] H1 Collab., I. Abt et al., Z. Phys. C 63, 377 (1994);

H1 Collab., S. Aid et al., Z. Phys. C 70, 609 (1996);

H1 Collab., C. Adloff et al., Eur. Phys. J. C 12, 595 (2000).

[12] T.Tymieniecka, Proc. of the 13th International Workshop on Deep Inelastic Scattering - DIS05, W.H. Smith and S.R. Dasu (ed.), p. 737. Madison, Wisconsin (2005).

[13] A.A. Sokolov and I.M. Ternov, Sov. Phys. Dokl. 8, 1203 (1964).

[14] ZEUS Collab., U. Holm (ed.), The ZEUS Detector. Status Report (unpublished), DESY (1993), available on http://www-zeus.desy.de/bluebook/bluebook.html.

[15] M. Derrick et al., Nucl. Instr. and Meth. A 309, 77 (1991);

A. Andresen et al., Nucl. Instr. and Meth. A 309, 101 (1991);

A. Caldwell et al., Nucl. Instr. and Meth. A 321, 356 (1992);

A. Bernstein et al., Nucl. Instr. and Meth. A 336, 23 (1993).

[16] N. Harnew et al., Nucl. Instr. and Meth. A 279, 290 (1989);

B. Foster et al., Nucl. Phys. Proc. Suppl. B 32, 181 (1993);

B. Foster et al., Nucl. Instr. and Meth. A 338, 254 (1994).

[17] S. Bentvelsen, J. Engelen and P. Kooijman, Proc. Workshop on Physics at HERA, W. Buchmüller and G. Ingelman (eds.), Vol. 1, p. 23. Hamburg, Germany, DESY (1992); 
K.C. Höger, Proc. Workshop on Physics at HERA, W. Buchmüller and G. Ingelman (eds.), Vol. 1, p. 43. Hamburg, Germany, DESY (1992).

[18] ZEUS Collab., J. Breitweg et al., Eur. Phys. J. C 6, 43 (1999);

G.M. Briskin, Ph.D. Thesis, Tel Aviv University, 1998. (Unpublished).

[19] R.D. Peccei and R. Rückl, Nucl. Phys. B 162, 125 (1980).

[20] G. Ingelman et al., Nucl. Phys. B 206, 239 (1982).

[21] R. Brun et al., GEAnt3, Technical Report CERN-DD/EE/84-1, CERN, 1987.

[22] G. Ingelman, A. Edin and J. Rathsman, Comp. Phys. Comm. 101, 108 (1997).

[23] A. Kwiatkowski, H. Spiesberger and H.-J. Möhring, Comp. Phys. Comm. 69, 155 (1992).

[24] H. Spiesberger, An Event Generator for ep Interactions at HERA Including Radiative Processes (Version 4.6), 1996, available on

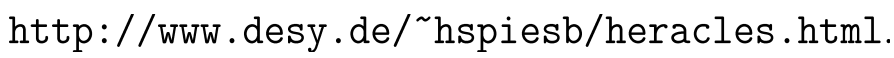

[25] H. Spiesberger, HERACLES and DJANGOH: Event Generation for ep Interactions at HERA Including Radiative Processes, 1998, available on http://www.desy.de/ ^hspiesb/djangoh.html.

[26] K. Charchula, G.A. Schuler and H. Spiesberger, Comp. Phys. Comm. 81, 381 (1994).

[27] L. Lönnblad, Comp. Phys. Comm. 71, 15 (1992).

[28] H.L. Lai et al., Phys. Rev. D 55, 1280 (1997).

[29] T. Sjöstrand and M. Bengtsson, Comp. Phys. Comm. 43, 367 (1987).

[30] A. Ukleja, Ph.D. Thesis, University of Warsaw, 2006. (Unpublished).

[31] S. Catani and M.H. Seymour, Nucl. Phys. B 485, 401 (1997).

[32] S. Catani and M.H. Seymour, Nucl. Phys. B 510, 503 (1998).

[33] R.K. Ellis, D.A. Ross and A.E. Terrano, Nucl. Phys. B 178, 421 (1981).

[34] J. Botts et al., Phys. Lett. B 304, 159 (1995).

[35] CTEQ Collab., H.L. Lai et al., Eur. Phys. J. C 12, 375 (2000).

[36] A.D. Martin et al., Eur. Phys. J. C 4, 463 (1998);

A.D. Martin et al., Eur. Phys. J. C 14, 133 (2000). 


\begin{tabular}{||c|ccc||}
\hline$\eta^{\mathrm{HCM}}$ & $\left\langle\cos \phi^{\mathrm{HCM}}\right\rangle$ & $\delta_{\text {stat }}$ & $\delta_{\text {syst }}$ \\
\hline \hline-4.75 & -0.034 & \pm 0.015 & ${ }_{-0.019}^{+0.009}$ \\
-4.25 & -0.064 & \pm 0.010 & ${ }_{-0.003}^{+0.016}$ \\
-3.75 & -0.062 & \pm 0.008 & ${ }_{-0.006}^{+0.004}$ \\
-3.25 & -0.066 & \pm 0.007 & ${ }_{-0.004}^{+0.008}$ \\
-2.75 & -0.068 & \pm 0.006 & ${ }_{-0.011}^{+0.008}$ \\
-2.25 & -0.030 & \pm 0.007 & ${ }_{-0.017}^{+0.005}$ \\
-1.75 & 0.010 & \pm 0.008 & ${ }_{-0.013}^{+0.002}$ \\
-1.25 & 0.020 & \pm 0.008 & ${ }_{-0.012}^{+0.002}$ \\
-0.75 & 0.028 & \pm 0.010 & ${ }_{-0.007}^{+0.002}$ \\
-0.25 & 0.019 & \pm 0.010 & ${ }_{-0.004}^{+0.012}$ \\
\hline
\end{tabular}

\begin{tabular}{||c|ccc||}
\hline$\eta^{\mathrm{HCM}}$ & $\left\langle\cos 2 \phi^{\mathrm{HCM}}\right\rangle$ & $\delta_{\text {stat }}$ & $\delta_{\text {syst }}$ \\
\hline \hline-4.75 & -0.011 & \pm 0.015 & ${ }_{-0.002}^{+0.017}$ \\
-4.25 & -0.019 & \pm 0.010 & ${ }_{-0.003}^{+0.008}$ \\
-3.75 & -0.029 & \pm 0.008 & ${ }_{-0.002}^{+0.013}$ \\
-3.25 & 0.009 & \pm 0.007 & ${ }_{-0.012}^{+0.000}$ \\
-2.75 & 0.004 & \pm 0.007 & ${ }_{-0.006}^{+0.006}$ \\
-2.25 & 0.015 & \pm 0.007 & ${ }_{-0.018}^{+0.004}$ \\
-1.75 & 0.025 & \pm 0.008 & ${ }_{-0.007}^{+0.009}$ \\
-1.25 & 0.028 & \pm 0.008 & ${ }_{-0.008}^{+0.006}$ \\
-0.75 & 0.030 & \pm 0.009 & ${ }_{-0.004}^{+0.004}$ \\
-0.25 & 0.004 & \pm 0.010 & ${ }_{-0.005}^{+0.006}$ \\
\hline
\end{tabular}

Table 1: The values of $\left\langle\cos \phi^{\mathrm{HCM}}\right\rangle$ and $\left\langle\cos 2 \phi^{\mathrm{HCM}}\right\rangle$, calculated using the energyflow method as in Eq. (2), as a function of hadron pseudorapidity, $\eta^{\mathrm{HCM}}$. They were obtained in the HCM frame for the kinematic region $100<Q^{2}<8000 \mathrm{GeV}^{2}$, $0.01<x<0.1$ and $0.2<y<0.8$ for hadrons with $p_{T}>0.15 \mathrm{GeV}$ and $\theta>8^{\circ}$. The quantities $\delta_{\text {stat }}$ and $\delta_{\text {syst }}$ are respectively the statistical and systematic uncertainties. 


\begin{tabular}{||c|ccc||}
\hline$\eta^{\mathrm{HCM}}$ & $\left\langle\sin \phi^{\mathrm{HCM}}\right\rangle$ & $\delta_{\text {stat }}$ & $\delta_{\text {syst }}$ \\
\hline \hline-4.75 & -0.007 & \pm 0.015 & ${ }_{-0.017}^{+0.006}$ \\
-4.25 & -0.018 & \pm 0.010 & ${ }_{-0.014}^{+0.005}$ \\
-3.75 & -0.023 & \pm 0.008 & ${ }_{-0.002}^{+0.004}$ \\
-3.25 & -0.016 & \pm 0.007 & ${ }_{-0.002}^{+0.004}$ \\
-2.75 & -0.001 & \pm 0.007 & ${ }_{-0.004}^{+0.002}$ \\
-2.25 & -0.012 & \pm 0.007 & ${ }_{-0.001}^{+0.002}$ \\
-1.75 & 0.009 & \pm 0.008 & ${ }_{-0.005}^{+0.001}$ \\
-1.25 & -0.003 & \pm 0.008 & ${ }_{-0.001}^{+0.006}$ \\
-0.75 & -0.018 & \pm 0.009 & ${ }_{-0.004}^{+0.006}$ \\
-0.25 & -0.025 & \pm 0.010 & ${ }_{-0.006}^{+0.003}$
\end{tabular}

\begin{tabular}{||c|ccc||}
\hline$\eta^{\mathrm{HCM}}$ & $\left\langle\sin 2 \phi^{\mathrm{HCM}}\right\rangle$ & $\delta_{\text {stat }}$ & $\delta_{\text {syst }}$ \\
\hline \hline-4.75 & 0.012 & \pm 0.015 & ${ }_{-0.002}^{+0.010}$ \\
-4.25 & -0.004 & \pm 0.010 & ${ }_{-0.007}^{+0.001}$ \\
-3.75 & 0.006 & \pm 0.008 & ${ }_{-0.007}^{+0.001}$ \\
-3.25 & -0.009 & \pm 0.007 & ${ }_{-0.002}^{+0.005}$ \\
-2.75 & -0.010 & \pm 0.007 & ${ }_{-0.006}^{+0.004}$ \\
-2.25 & 0.005 & \pm 0.007 & ${ }_{-0.003}^{+0.002}$ \\
-1.75 & -0.009 & \pm 0.008 & ${ }_{-0.002}^{+0.014}$ \\
-1.25 & -0.001 & \pm 0.008 & ${ }_{-0.007}^{+0.006}$ \\
-0.75 & 0.007 & \pm 0.010 & ${ }_{-0.005}^{+0.003}$ \\
-0.25 & 0.010 & \pm 0.010 & ${ }_{-0.002}^{+0.005}$ \\
\hline
\end{tabular}

Table 2: The values of $\left\langle\sin \phi^{\mathrm{HCM}}\right\rangle$ and $\left\langle\sin 2 \phi^{\mathrm{HCM}}\right\rangle$, calculated using the energyflow method as in Eq. (2), as a function of hadron pseudorapidity, $\eta^{\mathrm{HCM}}$. They were obtained in the HCM frame for the kinematic region $100<Q^{2}<8000 \mathrm{GeV}^{2}$, $0.01<x<0.1$ and $0.2<y<0.8$ for hadrons with $p_{T}>0.15 \mathrm{GeV}$ and $\theta>8^{\circ}$. The quantities $\delta_{\text {stat }}$ and $\delta_{\mathrm{syst}}$ are respectively the statistical and systematic uncertainties. 


\begin{tabular}{|c|c|c|c|}
\hline$E_{T, \text { min }}^{\mathrm{HCM}}(\mathrm{GeV})$ & $\left\langle\cos \phi^{\mathrm{HCM}}\right\rangle$ & $\delta_{\text {stat }}$ & $\delta_{\text {syst }}$ \\
\hline 0.0 & -0.064 & \pm 0.004 & $\begin{array}{l}+0.004 \\
{ }_{-0.005}\end{array}$ \\
\hline 0.5 & -0.074 & \pm 0.005 & ${ }_{-0.003}^{+0.006}$ \\
\hline 1.0 & -0.090 & \pm 0.007 & ${ }_{-0.003}^{+0.011}$ \\
\hline 1.5 & -0.099 & \pm 0.009 & $\begin{array}{l}{ }_{-0.007}^{+0.013} \\
\text { - }\end{array}$ \\
\hline 2.0 & -0.108 & \pm 0.011 & $\begin{array}{l}+0.011 \\
{ }_{-0.004}\end{array}$ \\
\hline 2.5 & -0.123 & \pm 0.013 & ${ }_{-0.004}^{+0.015}$ \\
\hline 3.0 & -0.128 & \pm 0.016 & $\begin{array}{l}+0.021 \\
{ }_{-0.007}\end{array}$ \\
\hline 3.5 & -0.131 & \pm 0.019 & ${ }_{-0.010}^{+0.020}$ \\
\hline 4.0 & -0.126 & \pm 0.023 & $\begin{array}{l}{ }_{-0.008}^{+0.019} \\
\end{array}$ \\
\hline
\end{tabular}

\begin{tabular}{|c|c|c|c|}
\hline$E_{T, \text { min }}^{\mathrm{HCM}}(\mathrm{GeV})$ & $\left\langle\cos \phi^{\mathrm{HCM}}\right\rangle$ & $\delta_{\text {stat }}$ & $\delta_{\text {syst }}$ \\
\hline 0.0 & 0.000 & \pm 0.004 & $\begin{array}{l}+0.002 \\
{ }_{-0.012}\end{array}$ \\
\hline 0.5 & 0.018 & \pm 0.006 & $\begin{array}{l}+0.003 \\
-0.007\end{array}$ \\
\hline 1.0 & 0.020 & \pm 0.008 & $\begin{array}{l}+0.004 \\
-0.008\end{array}$ \\
\hline 1.5 & 0.017 & \pm 0.010 & $\begin{array}{l}{ }_{-0.010}^{+0.004} \\
-0.00\end{array}$ \\
\hline 2.0 & 0.017 & \pm 0.011 & $\begin{array}{l}+0.005 \\
{ }_{-0.012}\end{array}$ \\
\hline 2.5 & 0.020 & \pm 0.013 & $\begin{array}{l}+0.005 \\
-0.014\end{array}$ \\
\hline 3.0 & 0.018 & \pm 0.015 & $\begin{array}{l}{ }_{-0.015}^{+0.005} \\
\end{array}$ \\
\hline
\end{tabular}

\begin{tabular}{|c|c|c|c|}
\hline \multicolumn{4}{|l|}{$-1<\eta^{\mathrm{HCM}} \leq 0$} \\
\hline$E_{T, \text { min }}^{\mathrm{HCM}}(\mathrm{GeV})$ & $\left\langle\cos \phi^{\mathrm{HCM}}\right\rangle$ & $\delta_{\text {stat }}$ & $\delta_{\text {syst }}$ \\
\hline 0.0 & 0.024 & \pm 0.007 & $\begin{array}{l}{ }_{-0.004}^{+0.004} \\
\end{array}$ \\
\hline 0.5 & 0.037 & \pm 0.010 & ${ }_{-0.003}^{+0.004}$ \\
\hline 1.0 & 0.042 & \pm 0.013 & $\begin{array}{l}+0.005 \\
{ }_{-0.005}\end{array}$ \\
\hline 1.5 & 0.046 & \pm 0.016 & $\begin{array}{l}+0.007 \\
{ }_{-0.009}\end{array}$ \\
\hline 2.0 & 0.044 & \pm 0.019 & $\begin{array}{l}+0.009 \\
{ }_{-0.009}\end{array}$ \\
\hline
\end{tabular}

Table 3: The values of $\left\langle\cos \phi^{\mathrm{HCM}}\right\rangle$ calculated using the energy-flow method as in Eq. (2), as a function of hadron minimum transverse energy, $E_{T, m i n}^{H C M}$. They were obtained in the HCM frame for the pseudorapidity intervals $-5<$ $\eta^{H C M} \leq-2.5,-2.5<\eta^{H C M} \leq-1$ and $-1<\eta^{H C M} \leq 0$ in the kinematic region $100<Q^{2}<8000 \mathrm{GeV}^{2}, 0.01<x<0.1$ and $0.2<y<0.8$ for hadrons with $p_{T}>0.15 \mathrm{GeV}$ and $\theta>8^{\circ}$. The quantities $\delta_{\text {stat }}$ and $\delta_{\text {syst }}$ are respectively the statistical and systematic uncertainties. 


\begin{tabular}{|c|c|c|c|}
\hline$E_{T, \min }^{\mathrm{HCM}}(\mathrm{GeV})$ & $\left\langle\cos 2 \phi^{\mathrm{HCM}}\right\rangle$ & $\delta_{\text {stat }}$ & $\delta_{\text {syst }}$ \\
\hline 0.0 & -0.002 & \pm 0.004 & $\begin{array}{l}+0.003 \\
-0.003\end{array}$ \\
\hline 0.5 & 0.002 & \pm 0.005 & $\begin{array}{l}+0.003 \\
-0.002\end{array}$ \\
\hline 1.0 & 0.006 & \pm 0.007 & $\begin{array}{l}+0.002 \\
-0.002\end{array}$ \\
\hline 1.5 & 0.009 & \pm 0.009 & $\begin{array}{l}+0.002 \\
-0.010\end{array}$ \\
\hline 2.0 & 0.016 & \pm 0.011 & $\begin{array}{l}+0.001 \\
-0.018\end{array}$ \\
\hline 2.5 & 0.014 & \pm 0.014 & ${ }_{-0.016}^{+0.003}$ \\
\hline 3.0 & 0.014 & \pm 0.017 & ${ }_{-0.028}^{+0.004}$ \\
\hline 3.5 & 0.019 & \pm 0.021 & ${ }_{-0.010}^{+0.005}$ \\
\hline 4.0 & 0.006 & \pm 0.025 & ${ }_{-0.005}^{+0.012}$ \\
\hline
\end{tabular}

\begin{tabular}{|c|c|c|c|}
\hline \multicolumn{4}{|c|}{$-2.5<\eta^{\mathrm{HCM}} \leq-1$} \\
\hline$E_{T, \min }^{\mathrm{HCM}}(\mathrm{GeV})$ & $\left\langle\cos 2 \phi^{\mathrm{HCM}}\right\rangle$ & $\delta_{\text {stat }}$ & $\delta_{\text {syst }}$ \\
\hline 0.0 & 0.022 & \pm 0.004 & $\begin{array}{l}+0.004 \\
-0.007\end{array}$ \\
\hline 0.5 & 0.039 & \pm 0.006 & $\begin{array}{l}+0.005 \\
-0.006\end{array}$ \\
\hline 1.0 & 0.055 & \pm 0.008 & $\begin{array}{l}{ }_{-0.009}^{+0.006} \\
\end{array}$ \\
\hline 1.5 & 0.062 & \pm 0.009 & $\begin{array}{l}+0.007 \\
-0.011\end{array}$ \\
\hline 2.0 & 0.067 & \pm 0.011 & $\begin{array}{l}+0.008 \\
-0.010\end{array}$ \\
\hline 2.5 & 0.070 & \pm 0.012 & ${ }_{-0.010}^{+0.009}$ \\
\hline 3.0 & 0.076 & \pm 0.014 & $\begin{array}{l}+0.010 \\
{ }_{-0.011}\end{array}$ \\
\hline
\end{tabular}

\begin{tabular}{|c|c|c|c|}
\hline \multicolumn{4}{|c|}{$-1<\eta^{\mathrm{HCM}} \leq 0$} \\
\hline$E_{T, \min }^{\mathrm{HCM}}(\mathrm{GeV})$ & $\left\langle\cos 2 \phi^{\mathrm{HCM}}\right\rangle$ & $\delta_{\text {stat }}$ & $\delta_{\text {syst }}$ \\
\hline 0.0 & 0.018 & \pm 0.007 & $\begin{array}{l}+0.005 \\
-0.004\end{array}$ \\
\hline 0.5 & 0.022 & \pm 0.009 & ${ }_{-0.005}^{+0.005}$ \\
\hline 1.0 & 0.026 & \pm 0.013 & $\begin{array}{l}+0.008 \\
{ }_{-0.008}\end{array}$ \\
\hline 1.5 & 0.032 & \pm 0.016 & ${ }_{-0.010}^{+0.010}$ \\
\hline 2.0 & 0.034 & \pm 0.018 & $\begin{array}{l}+0.012 \\
{ }_{-0.013}\end{array}$ \\
\hline
\end{tabular}

Table 4: The values of $\left\langle\cos \phi^{\mathrm{HCM}}\right\rangle$ calculated using the energy-flow method as in Eq. (2), as a function of hadron minimum transverse energy, $E_{T, m i n}^{H C M}$. They were obtained in the HCM frame for the pseudorapidity intervals $-5<$ $\eta^{H C M} \leq-2.5,-2.5<\eta^{H C M} \leq-1$ and $-1<\eta^{H C M} \leq 0$ in the kinematic region $100<Q^{2}<8000 \mathrm{GeV}^{2}, 0.01<x<0.1$ and $0.2<y<0.8$ for hadrons with $p_{T}>0.15 \mathrm{GeV}$ and $\theta>8^{\circ}$. The quantities $\delta_{\text {stat }}$ and $\delta_{\text {syst }}$ are respectively the statistical and systematic uncertainties. 


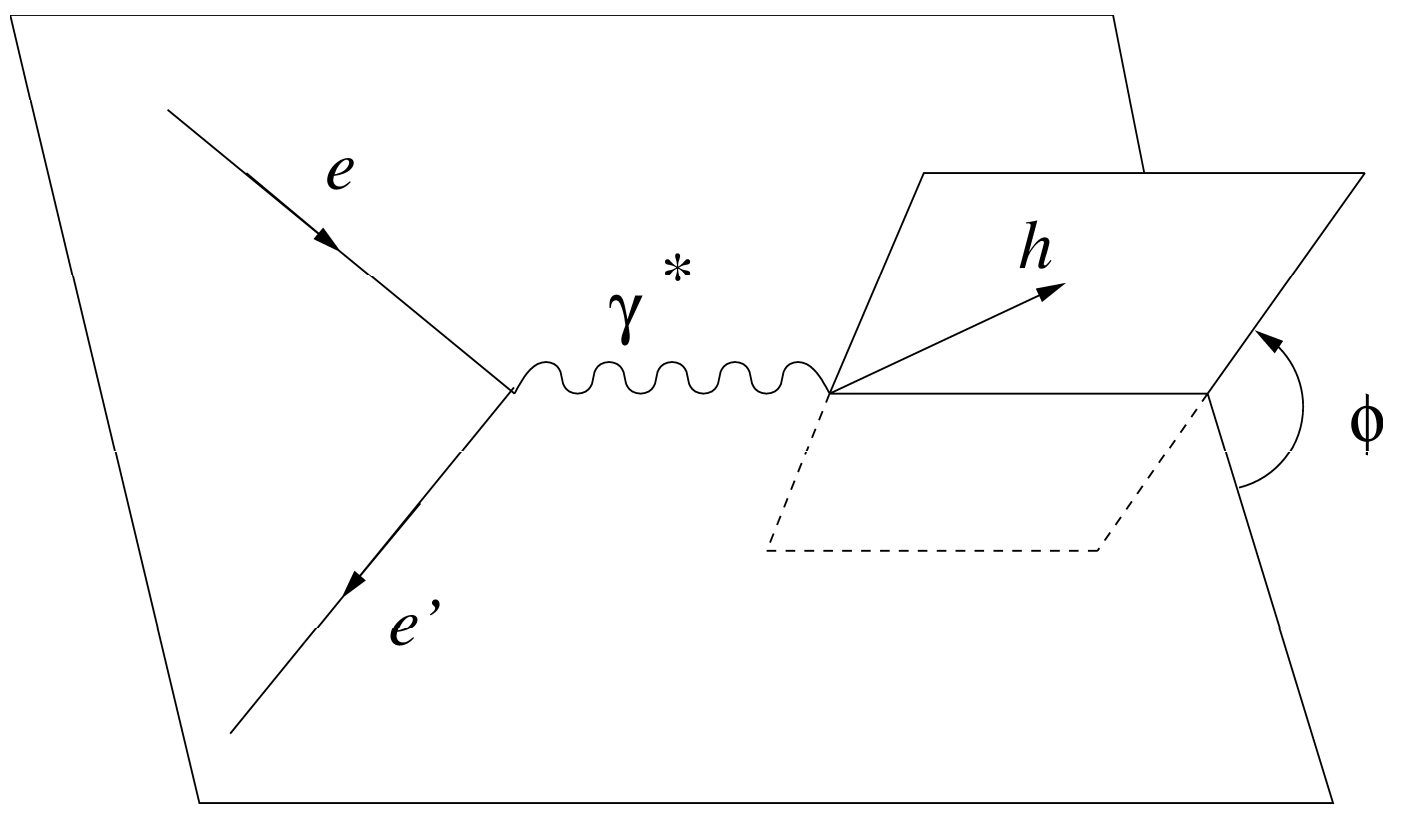

Figure 1: The definition of the azimuthal angle $\phi$ either in the $H C M$ or the Breit frame. The incoming electron is denoted by e, the scattered electron by $e^{\prime}$, the exchanged virtual photon by $\gamma^{*}$ and the outgoing hadron or parton by $h$. 

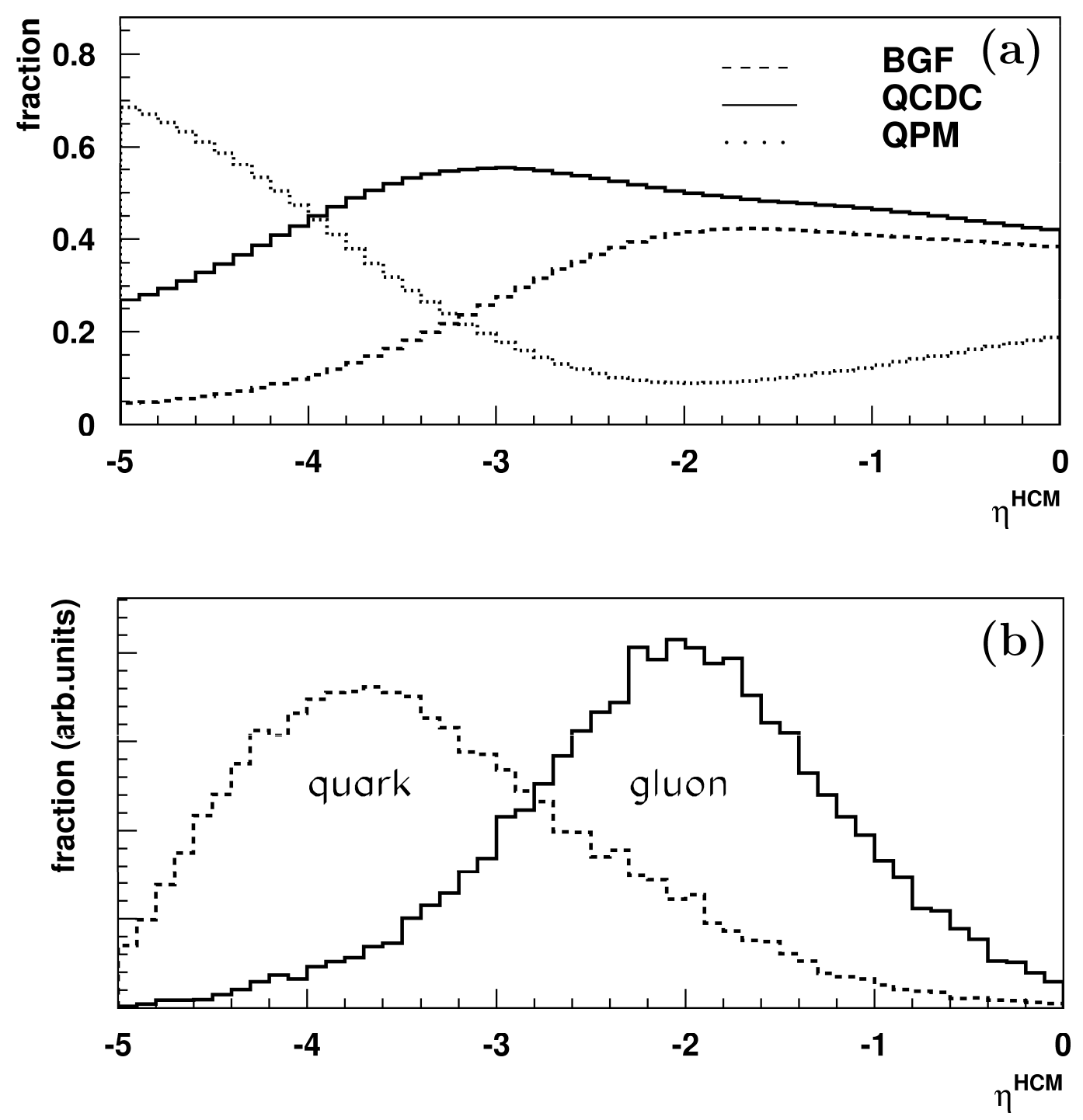

Figure 2: (a) The fraction of BGF (dashed line), QCDC (full line) and QPM (dotted line) processes as a function of pseudorapidity, $\eta^{H C M}$, in the HCM frame for the energy-flow method. (b) For the QCD Compton process, the quark and gluon contributions as a function of $\eta^{H C M}$. These predictions were taken from LEPTO 6.5.1 and are shown for the kinematic region $100<Q^{2}<8000 \mathrm{GeV}^{2}, 0.2<y<0.8$ and $0.01<x<0.1$ for hadrons with $p_{T}>0.15 \mathrm{GeV}$ and $\theta>8^{\circ}$. 


\section{ZEUS}
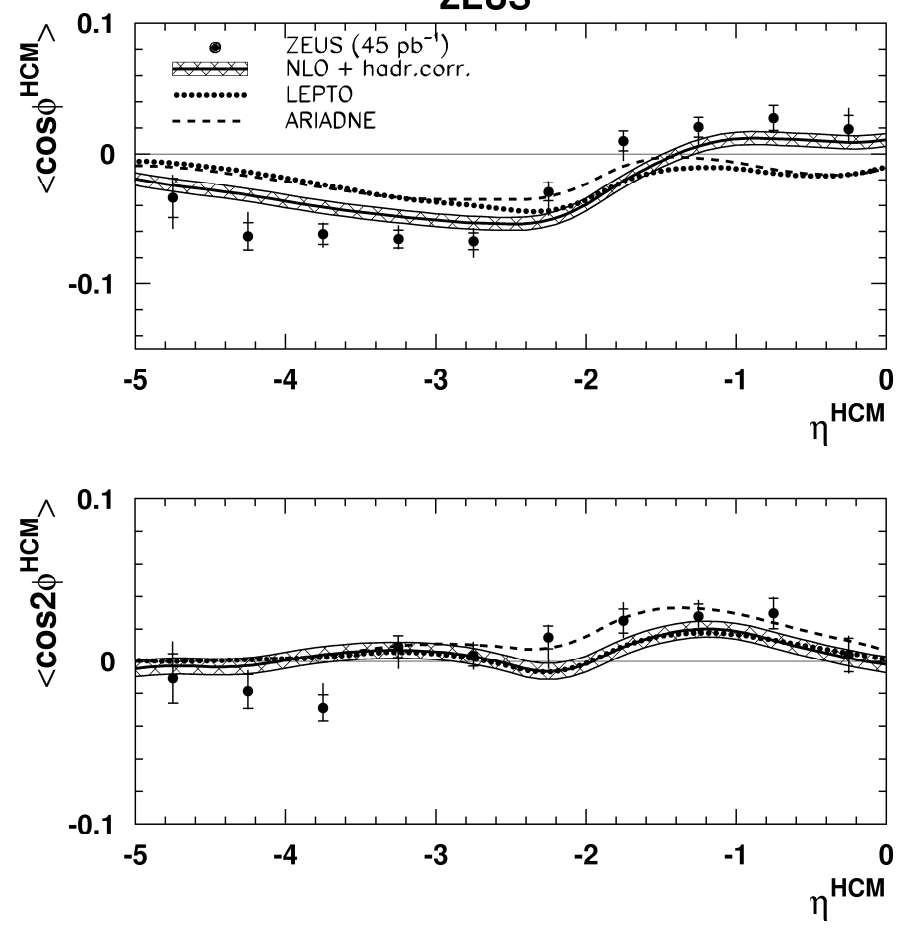

ZEUS
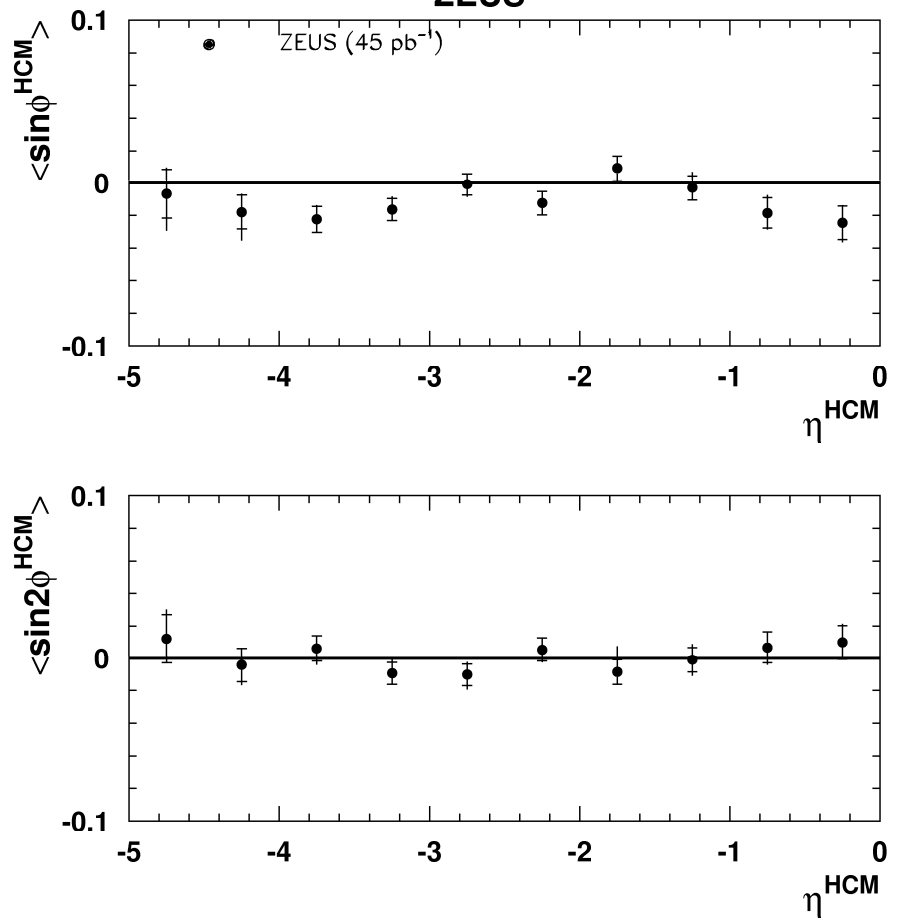

Figure 3: The values of $\left\langle\cos \phi^{H C M}\right\rangle,\left\langle\cos 2 \phi^{H C M}\right\rangle,\left\langle\sin \phi^{H C M}\right\rangle$ and $\left\langle\sin 2 \phi^{H C M}\right\rangle$, calculated using the energy-flow method as in Eq. (2), as a function of hadron pseudorapidity, $\eta^{H C M}$. They were obtained in the HCM frame for the kinematic region $100<Q^{2}<8000 \mathrm{GeV}^{2}, 0.01<x<0.1$ and $0.2<y<0.8$ for hadrons with $p_{T}>0.15 \mathrm{GeV}$ and $\theta>8^{\circ}$. The inner error bars are statistical uncertainties, the outer are statistical and systematic uncertainties added in quadrature. The NLO QCD predictions of DISENT (solid line), with its associated uncertainty (shaded band), corrected for hadronisation and hadron losses (see text), the predictions of LEPTO 6.5.1 (dotted line), and the predictions of ARIADNE 4.12 (dashed line) are shown. 


\section{ZEUS}
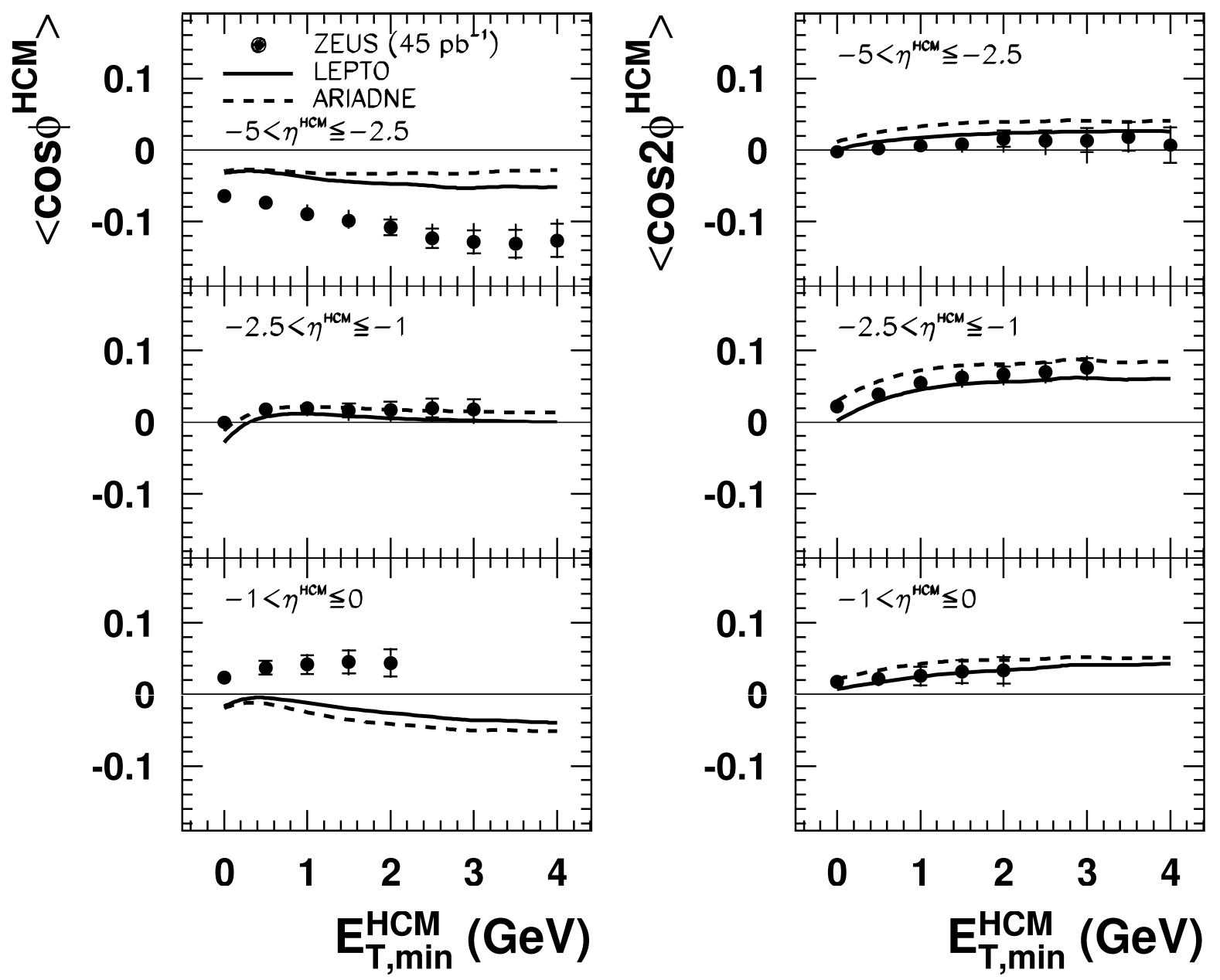

Figure 4: The values of $\left\langle\cos \phi^{H C M}\right\rangle$ and $\left\langle\cos 2 \phi^{H C M}\right\rangle$, calculated using the energyflow method as in Eq. (2), as a function of hadron minimum transverse energy, $E_{T, \text { min }}^{H C M}$. They were obtained in the $H C M$ frame for the pseudorapidity intervals $-5<\eta^{H C M} \leq-2.5,-2.5<\eta^{H C M} \leq-1$ and $-1<\eta^{H C M} \leq 0$ in the kinematic region $100<Q^{2}<8000 \mathrm{GeV}^{2}, 0.01<x<0.1$ and $0.2<y<0.8$ for hadrons with $p_{T}>0.15 \mathrm{GeV}$ and $\theta>8^{\circ}$. The inner error bars are statistical uncertainties, the outer are statistical and systematic uncertainties added in quadrature. The predictions of LEPTO 6.5 .1 (solid line) and of ARIADNE 4.12 (dashed line) are shoun. 